\title{
Plasmon-enhanced magneto-optical activity in ferromagnetic membranes
}

\author{
J. B. González-Díaz, ${ }^{1}$ J. M. García-Martín, ${ }^{1, a)}$ A. García-Martín, ${ }^{1}$ D. Navas, ${ }^{2,3}$ A. Asenjo, ${ }^{2}$ \\ M. Vázquez, ${ }^{2}$ M. Hernández-Vélez, ${ }^{4}$ and G. Armelles ${ }^{1}$ \\ ${ }^{1}$ Instituto de Microelectrónica de Madrid (IMM-CNM-CSIC), Isaac Newton 8, Tres Cantos, \\ 28760 Madrid, Spain \\ ${ }^{2}$ Instituto de Ciencia de Materiales de Madrid (ICMM-CSIC), Sor Juana Inés de la Cruz 3, Cantoblanco, \\ 28049 Madrid, Spain \\ ${ }^{3}$ Department of Materials Science and Engineering, MIT, Cambridge, Massachussetts 02139, USA \\ ${ }^{4}$ Departamento de Física Aplicada, Universidad Autónoma de Madrid, Cantoblanco, 28049 Madrid, Spain
}

(Received 17 April 2009; accepted 11 June 2009; published online 30 June 2009)

\begin{abstract}
Experimental and theoretical evidence of plasmon-enhanced Kerr rotation in purely ferromagnetic membranes with sufficiently small dimensions to be out of extraordinary optical transmission conditions (45 $\mathrm{nm}$ pore diameter, $90 \mathrm{~nm}$ lattice constant), is reported in this work. It is shown that the spectral location of the enhanced Kerr rotation region varies as the refractive index of the material inside the pore is modified. A similar behavior is obtained if the pore radius changes while keeping the pore concentration unchanged. Those are clear signatures indicating that localized surface plasmon resonances propagating along the pores govern the magneto-optical response of the membrane. (c) 2009 American Institute of Physics. [DOI: 10.1063/1.3167297]
\end{abstract}

Nanoholed ferromagnetic membranes are attracting a great deal of interest over the last years due to their unique magnetic behavior. ${ }^{1-6}$ Their magnetic properties are strongly influenced by the precise geometry of the nanostructure, namely the pore diameter and the interpore distance since these parameters control the nucleation and movement of domain walls as well as induce locally distributed magnetic anisotropies absent in unpatterned films. ${ }^{7,8}$ From an applied viewpoint, ferromagnetic membranes can be used for developing data storage media based on domain wall structures as well as advanced sensing devices. ${ }^{1,2,9}$

On a different context, membranes made of noble metals have been deeply studied since they exhibit anomalous optical responses resulting from the excitation of plasmons in the nanostructures, such as the extraordinary optical transmission (EOT) bands observed in metallic patterned thin films with arrays of subwavelength holes. ${ }^{10}$ There have been attempts to control this EOT using liquid crystals ${ }^{11}$ or exploiting the intrinsic magneto-optical (MO) activity present in every metal, ${ }^{12,13}$ although this would require huge magnetic fields for the nonferromagnetic membranes. Belotelov et al. ${ }^{14}$ have used these extraordinary effects to modify the response of nonstructured $\mathrm{MO}$ materials, e.g., using Au film with subwavelength holes array deposited on top of a Bismuth substituted Yttrium Iron garnet (BiYIG) film. In the spectral region where the EOT takes place, the MO response (Kerr signal) shows an increase with respect to that of an unpatterned film, which is attributed to the purely optical effect of strong decrease of the reflectance.

Ferromagnetic nanostructures have shown interesting optical and MO properties related to the excitation of surface plasmons as shown in ordered arrays of Ni nanowires embedded in an alumina matrix. ${ }^{15,16}$ In these nanostructures, the plasmons, propagating along the wires, were responsible of the observed MO response enhancement that has not merely optical origin. Diwekar et al. ${ }^{17}$ observed the existence of EOT bands in Co films with subwavelength hole arrays (150

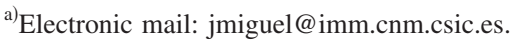

$\mathrm{nm}$ diameter, $300 \mathrm{~nm}$ periodicity), which was explained as due to light coupling to surface plasmons on the two film interfaces. Quite recently, Ctistis et al. ${ }^{18}$ have studied the MO response of different Co membranes (diameter between 220 and $330 \mathrm{~nm}$, the lattice constant always being $470 \mathrm{~nm}$ ) and it has been compared to that of a continuous film. The observed enhanced response of the Co membranes has been attributed to surface plasmon excitations.

In this work, we study the $\mathrm{MO}$ activity of $\mathrm{Ni}$ membranes in the spectral range from 280 to $850 \mathrm{~nm}$. Despite the fact that no EOT band are observed, there is an enhancement of the MO activity in the UV range, which we attribute to localized surface plasmon resonance of the pores. We provide both experimental evidences (such as the dependence of the MO response with the reactive index $n$ of the material filling the pores) and theoretical simulations supporting this assessment.

The Ni membranes are prepared by replica/antireplica methods using nanoporous anodic alumina membranes as templates. ${ }^{4}$ Note that the diameter and the lattice constant are similar to those of $\mathrm{Ni}$ nanowires embedded in $\mathrm{Al}_{2} \mathrm{O}_{3}$ studied previously, ${ }^{15,16}$ so allowing a direct comparison. Therefore, we compare the magneto-optic behavior of three different samples: (a) Ni membrane with $d=45 \mathrm{~nm}$ and $a=90 \mathrm{~nm}$, (b) Ni nanowires array with very close geometrical parameters $(d=40 \mathrm{~nm}$ and $a=105 \mathrm{~nm})$, and (c) Ni bulk.

To obtain the ferromagnetic membranes by replica/ antireplica methods, Au thin layer, 15-nm-thick, is sputtered on top of the alumina membrane and the pores are filled in with melted polymethyl methacrylate (PMMA) by pressure. Removing both $\mathrm{Al}$ substrate and alumina membrane by chemical etchings, a PMMA antireplica of the alumina membrane (i.e., an array of pillars) is obtained. Thanks to the Au electrode previously deposited, a Ni membrane can be fabricated by electrodeposition. Finally, the PMMA pillars are chemically removed. Morphological characterization of the Ni membrane was carried out by scanning electron microscopy (SEM). Figure 1 shows SEM images of the Ni (top) 


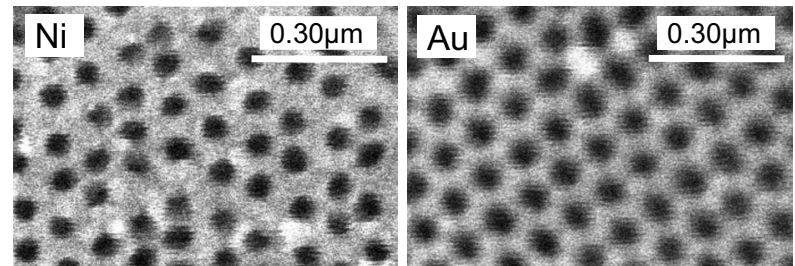

FIG. 1. Scanning electron micrographs of both sides of a representative membrane obtained by the replica/antireplica method.

and $\mathrm{Au}$ (bottom) sides of a representative membrane $200 \mathrm{~nm}$ thick.

In order to analyze the MO response, we have measured the Kerr spectra in polar configuration, i.e., applying a magnetic field perpendicular to the sample plane and measuring the rotation of the polarization plane of the reflected light. The measurements were performed at normal incidence. The intensity of the applied magnetic field, 1.6 T, was high enough to nearly saturate magnetically the samples. The resulting polar Kerr rotation spectrum is shown as filled circles in Fig. 2, where it is also depicted the spectra for Ni bulk (line) and the ordered array of $\mathrm{Ni}$ wires (open circles). As observed, the spectra show two different regions: the short wavelength (i.e., high energy) region where the spectra seem to be similar and the rotation reaches a minimum (a maximum in absolute value, the minus sign only means that the rotation is anticlockwise), and the long wavelength (i.e., low energy) region where the three spectra present a monotonous evolution and the rotation reaches different values.

The long wavelength region, where the rotation reaches different values for the three samples, is the more intuitive one: in absence of any resonance or other anomalous effect, the Kerr rotation is expected to be roughly proportional to the amount of ferromagnetic material in a first approximation. In a hexagonal array of holes with diameter $d$ and lattice constant $a$, the ratio between the area covered by the holes and the total surface is given by

$$
\left(\frac{\pi}{2 \sqrt{3}}\right) \times(d / a)^{2} .
$$

Taking into account the parameters of our samples, the amount of $\mathrm{Ni}$ in the nanowire array sample and in the membrane would be $13 \%$ and $77 \%$ of that of $\mathrm{Ni}$ bulk, respectively. From the values shown in Fig. 2 at about $800 \mathrm{~nm}$ wavelength, we can see that the Kerr rotation for the nanowire array and for the membrane are $14 \%$ and $63 \%$ of that of the continuous $\mathrm{Ni}$ film, respectively, i.e., in good qualitative

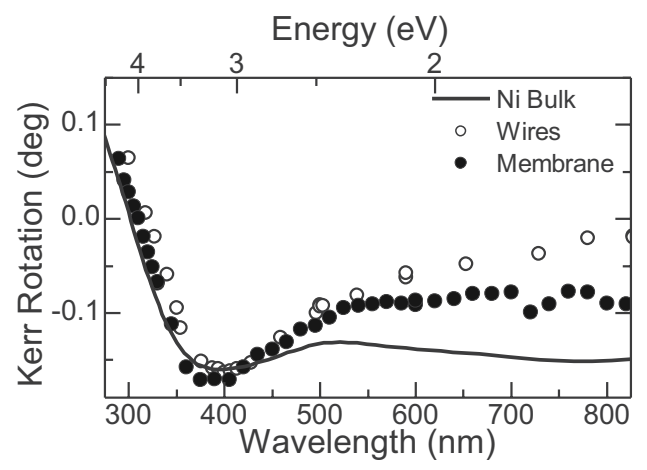

FIG. 2. Polar Kerr rotation spectra of the membrane in Fig. 1 (filled circles), a nanowire array (open circles), and Ni bulk (continuous line).

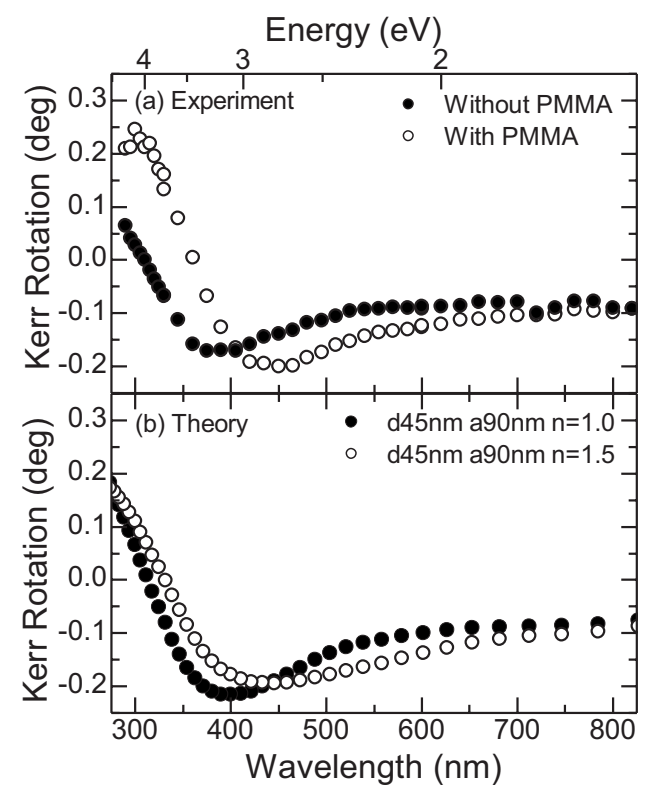

FIG. 3. Experimental polar Kerr rotation spectra of the same membrane as in Figs. 1 and 2, before (open circles) and after (filled circles) the PMMA is removed. Simulated spectra of those membranes $(d=45 \mathrm{~nm}$ and $a=90 \mathrm{~nm})$.

agreement with the aforementioned expectations.

However, in the low wavelength region, the Kerr rotation signal does not follow this simple rule of thumb. In fact, a minimum of around $400 \mathrm{~nm}$ with similar Kerr rotation can be observed for the three samples. In the case of $\mathrm{Ni}$ nanowires, it has been demonstrated ${ }^{15,16}$ that the excitation of surface plasmons propagating along the wires provides an intensification of the electromagnetic (EM) field compensating the smaller amount of MO active material when compared to the bulk, therefore leading to similar Kerr rotation values. The similar behavior observed in the Ni membranes suggests a similar mechanism for the enhancement. In particular, the enhancement should be related to the excitation of plasmons propagating along the pores. When the surface plasmon is excited there is an enhancement of the EM field in the Ni regions around the pore and therefore an increase in the MO activity. If plasmon excitation can be regarded as responsible of such enhancement in the MO response of the membrane, then the spectral location of the maximum Kerr rotation must vary as the refractive index $n$ of the material inside the pore is modified, since the plasmon characteristics would be altered. The fabrication method used to prepare the membranes provides the means to obtain an experimental verification of the redshift originated on the increase in the infilling $n$ : by stopping in the preparation process before the last step, a Ni membrane with PMMA inside the pores can be obtained. In Fig. 3(a), we present the Kerr rotation of the same $\mathrm{Ni}$ membrane as in Figs. 1 and 2, but before (open circles) and after (filled circles) the PMMA (whose $n$ is higher than that of air) was chemically removed. As can be observed, the spectra are different. The differences are being more evident in the UV region, where a clear redshift of the structure is observed as the $n$ of the material filling the pores increases. This behavior is the expected one provided the enhancement is due to surface plasmons resonances of the pores: any increase in the $n$ of the material filling the pore makes the plasmon resonance shift toward lower energies. We have also performed theoretical calculations for mem- 


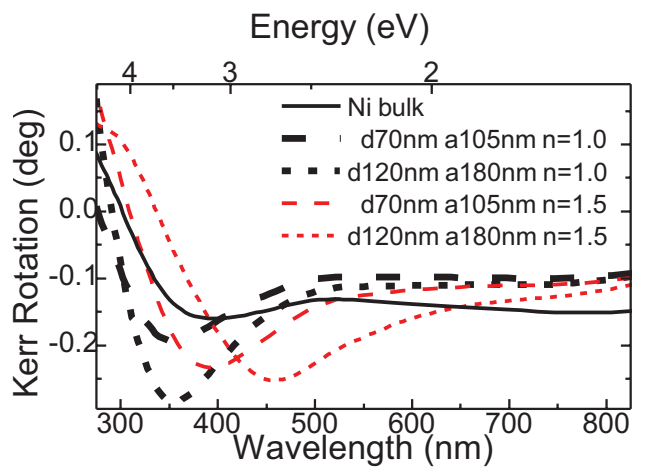

FIG. 4. (Color online) Theoretical polar Kerr rotation spectra of nickel bulk (continuous line) and four different membranes (pore-magnetic material ratio constant). Thick lines: $d=70 \mathrm{~nm}$ (dashed line) and $d=120 \mathrm{~nm}$ (dotted line) membranes with dielectric exhibiting $n=1.0$ filling the pores. Thin lines: same as thick lines but for a dielectric with $n=1.5$.

branes using a scattering matrix method modified to allow MO activity (a detailed description is given in Ref. 19). The optical and MO constants employed were obtained from Ref. 20. The geometrical parameters used in the calculations are the same of the experimental ones, and the pores were filled with different infilling materials (with $n=1$ and $n=1.5$, respectively) as shown in Fig. 3(b). As can be observed the simulation reproduces the enhancement of the MO activity in the UV region as well as the redshift observed in the experimental spectra due to the increase in the $n$ of the infilling material; nevertheless, the changes observed in the experimental spectra are more pronounced than the calculated ones. Such difference could be mainly due to an inadequate description of the optical properties of the material inside the pore.

For completeness, we present in Fig. 4 the calculated Kerr rotation spectra for structures, which have the same amount of $\mathrm{Ni}$ but different pore diameter, interpore distances, and different pores filling refractive index: two of them (A and B) with pore diameter $d=70 \mathrm{~nm}$ and lattice constant $a$ $=105 \mathrm{~nm}$ but different infilling $n \quad(n=1$ and $n=1.5)$, and other two (C and D) with $d=120 \mathrm{~nm}$ and $a=180 \mathrm{~nm}$ (i.e., keeping constant the pore-magnetic material ratio) also with $n=1$ and $n=1.5$, respectively. In the long wavelength region, where no plasmon exists, all the simulated membranes exhibit similar Kerr rotation value, proportional to the amount of nickel $(60 \%$ of the bulk). However, in the short wavelength region, a redshift of the spectra of $\mathrm{C}$ and $\mathrm{D}$ is observed with respect to those of $\mathrm{A}$ and $\mathrm{B}$, respectively, which is ascribed to the increase in the diameter. This redshift is precisely what is expected if plasmons propagating along the walls of the pores are excited. Also, a redshift of the spectra of $\mathrm{B}$ and $\mathrm{D}$ with respect to those of $\mathrm{A}$ and $\mathrm{C}$, respectively, is observed due to the increase in the infilling refractive index, as previously explained for Fig. 3. Moreover, similar to the behavior found in the experimental results, an enhancement of the MO rotation can be appreciated with respect to the nickel bulk in all the cases, pointing out the effect of the plasmon excitation on the MO activity.

In summary, we have presented experimental and theoretical evidence of plasmon-enhanced Kerr rotation in purely ferromagnetic membranes, out of EOT conditions. We have demonstrated that, in the plasmon excitation region, membranes as well as nanowire arrays present similar Kerr rotation intensities, whereas in the other spectral region, where no plasmon is excited, the intensity of the rotation turns out to be basically proportional to the amount of ferromagnetic material. We have also demonstrated that the spectral position of the enhancement can be tuned by an adequate choice of the refractive index of the material filling the pores and/or geometrical parameters.

We acknowledge Spanish MICINN (Grant Nos. MAT2008-06765-C02-01/NAN, CSD2008-00023, and MAT2007-65420-C02-01), CSIC (Grant No. PIF 200560F0121 BIOPTOMAG), CM (Grant No. S-0505/MAT/ 0194 NANOMAGNET), and European Commission (Grant No. NMP3-SL-2008-214107-Nanomagma) for financial support.

${ }^{1}$ Z. L. Xiao, C. Y. Han, U. Welp, H. H. Wang, V. K. Vlasko-Vlasov, W. K. Kwok, D. J. Miller, J. M. Hiller, R. E. Cook, G. A. Willing, and G. W Crabtree, Appl. Phys. Lett. 81, 2869 (2002).

${ }^{2}$ R. P. Cowburn, A. O. Adeyeye, and J. A. C. Bland, Appl. Phys. Lett. 70, 2309 (1997).

${ }^{3}$ T. Yanagishita, K. Nishio, and H. Masuda, Adv. Mater. 17, 2241 (2005).

${ }^{4}$ D. Navas, M. Hernández-Vélez, M. Vázquez, W. Lee, and K. Nielsch, Appl. Phys. Lett. 90, 192501 (2007).

${ }^{5}$ F. J. Castaño, K. Nielsch, C. A. Ross, J. W. A. Robinson, and R. Krishnan, Appl. Phys. Lett. 85, 2872 (2004).

${ }^{6}$ L. J. Heyderman, F. Nolting, D. Backes, S. Czekaj, L. Lopez-Diaz, M. Kläui, U. Rüdiger, C. A. F. Vaz, J. A. C. Bland, R. J. Matelon, U. G. Volkmann, and P. Fischer, Phys. Rev. B 73, 214429 (2006).

${ }^{7}$ D. Navas, M. Hernández-Vélez, A. Asenjo, M. Jaafar, J. L. Baldonedo, and M. Vázquez, IEEE Trans. Magn. 42, 3057 (2006).

${ }^{8}$ M. Jaafar, D. Navas, A. Asenjo, M. Vázquez, M. Hernández-Vélez, and J. M. García-Martín J. Appl. Phys. 101, 09F513 (2007).

${ }^{9}$ M. B. A. Jalil, J. Appl. Phys. 93, 7053 (2003).

${ }^{10}$ T. W. Ebbesen, H. J. Lezec, H. G. Ghaemi, T. Thio, and P. A. Wolff, Nature (London) 391, 667 (1998).

${ }^{11}$ Y. M. Strelniker, D. Stroud, and A. O. Voznesenskaya, Eur. Phys. J. B 52, 1 (2006)

${ }^{12}$ A. Battula, S. Chen, Y. Lu, R. J. Knize, and K. Reinhardt, Opt. Lett. 32, 2692 (2007)

${ }^{13}$ Y. M. Strelniker and D. J. Bergman, Phys. Rev. B 77, 205113 (2008).

${ }^{14}$ V. I. Belotelov, L. L. Doskolovich, and A. K. Zvezdin, Phys. Rev. Lett. 98, 077401 (2007).

${ }^{15}$ J. B. González-Díaz, A. García-Martín, G. Armelles, D. Navas, M. Vázquez, K. Nielsch, R. B. Wehrspohn, and U. Gösele, Adv. Mater. 19, 2643 (2007)

${ }^{16} \mathrm{~S}$. Melle, J. L. Menéndez, G. Armelles, D. Navas, M. Vázquez, K. Nielsch, R. B. Wehrspohn, and U. Gösele, Appl. Phys. Lett. 83, 4547 (2003).

${ }^{17}$ M. Diwekar, V. Kamaev, J. Shi, and Z. V. Vardeny, Appl. Phys. Lett. 84, 3112 (2004)

${ }^{18}$ G. Ctistis, E. Papaioannou, P. Patoka, J. Gutek, P. Fumagalli, and M. Giersig, Nano Lett. 9, 1 (2009).

${ }^{19}$ A. Garcia-Martin, G. Armelles, and S. Pereira, Phys. Rev. B 71, 205116 (2005).

${ }^{20}$ S. Visnovsky, V. Parizek, M. Nyvlt, P. Kielar, V. Prosser, and R. Krishman, J. Magn. Magn. Mater. 127, 135 (1993). 